\title{
Cherenkov Effect as a Probe of Photonic Nanostructures
}

\author{
F. J. García de Abajo, ${ }^{1}$ A. G. Pattantyus-Abraham, ${ }^{2}$ N. Zabala,,${ }^{1,3}$ A. Rivacoba, ${ }^{1,4}$ M. O. Wolf, ${ }^{2}$ and P. M. Echenique ${ }^{1,4}$ \\ ${ }^{1}$ Centro Mixto CSIC-UPV/EHU and Donostia International Physics Center (DIPC), San Sebastián, Spain \\ ${ }^{2}$ Department of Chemistry, University of British Columbia, Vancouver, Canada \\ ${ }^{3}$ Departamento de Electricidad y Electrónica, Facultad de Ciencias, UPV/EHU, Bilbao, Spain \\ ${ }^{4}$ Departamento de Física de Materiales, Facultad de Química, UPV/EHU, San Sebastián, Spain
}

(Received 8 May 2003; published 30 September 2003)

\begin{abstract}
Electron energy-loss spectroscopy (EELS) is shown to be an excellent source of information both on photonic crystal bands and on radiation modes of complex nanostructures. Good agreement is reported between measurements and parameter-free calculations of EELS in porous alumina films, where Cherenkov radiation is scattered by the pores to yield a strong $8.3-\mathrm{eV}$ (7-eV) feature for $120-\mathrm{keV}$ $(200-\mathrm{keV})$ electrons. The latter is related to the bands of two-dimensional photonic crystals formed by air cylinders in an alumina matrix with similar near-range ordering. Finally, the band structure is proved to be directly mapped by angle-resolved EELS.
\end{abstract}

DOI: 10.1103/PhysRevLett.91.143902

PACS numbers: 42.70.Qs, 41.60.Bq, 79.20.-m

Electron energy-loss spectroscopy (EELS) has been routinely used to determine optical properties of bulk materials and small particles $[1,2]$. This is usually performed in electron microscopes, where fast electrons $(20-300 \mathrm{keV})$ are energy analyzed after they traverse the sample. Energy losses are generally associated to creation of excitations in the target, although coupling to radiation modes can also lead to substantial effects. In particular, Cherenkov radiation (CR) losses arise when the electron velocity exceeds the speed of light in a transparent medium [3]. Furthermore, induced charges and currents near inhomogeneous systems are necessarily subject to acceleration, so that they give rise to radiative losses as well, as in the Smith-Purcell effect [4]. In periodically structured materials, CR can be emitted by subluminal electrons assisted by momentum transfer from the lattice and phonon creation [5], and in the related context of photonic crystals, the possibility of complicated Cherenkov light patterns has been recently reported [6].

In this Letter, the ability of EELS to probe radiation modes in complex nanostructures is demonstrated using $120-\mathrm{keV}(200-\mathrm{keV})$ electrons on porous alumina. A prominent loss peak in the poorly absorbing region at $\approx 8.3 \mathrm{eV}(\approx 7 \mathrm{eV})$ is successfully explained in terms of coupling to confined CR modes. This feature is then connected to the band structure of two-dimensional (2D) photonic crystals formed by infinitely long cylindrical holes drilled in alumina, for which the loss spectra are shown to follow the main features of the photonic density of states (DOS). Further details of the bands are proved to be retrievable by resolving momentum transfers perpendicular to the electron trajectory.

Porous alumina films were prepared by $\mathrm{Al}$ anodization [7]. A typical sample is shown in Fig. 1(a). Using a scanning transmission electron microscope (STEM) with a field-emission source at 200-keV nominal energy, a 2-nm electron beam was transmitted through one of the pores with three different impact parameters [Fig. 1(b)] for which loss spectra were collected separately, as shown in Fig. 1(c) (symbols). Further details on sample preparation and data acquisition will be given elsewhere [8]. All three spectra exhibit a feature near $7 \mathrm{eV}$ that is partially shadowed by higher-energy, surface plasmon losses as the beam approaches the pore walls. This peak lies in a region where alumina is almost nonabsorbing $(\epsilon=$ $3.76+0.03 i$ ) for the sample dimensions under consideration, and, therefore, it must originate in the coupling of sample radiation modes to these nondestructive aloof trajectories that do not touch the material.

A theoretical discussion of this feature is offered in Fig. 2(a) for axial trajectories of $200-\mathrm{keV}$ electrons in a pore surrounded by different structures. Loss spectra have been calculated from the retarding force exerted on the electron by its induced electric field, which is in turn obtained from Maxwell's equations by means of the boundary element method for arbitrarily shaped objects [9]. The frequency-dependent dielectric function of alumina has been taken from optical data [1]. The isolated pore (dashed curve) produces a featureless spectrum due to CR that leaks away from the pore. In a naive picture, the effect of neighboring pores is contained to some extent in a cylindrical shell with outer radius equal to the average interpore separation $\approx 90 \mathrm{~nm}$ (dash-dotted curve). This leads to a peak near $7 \mathrm{eV}$ as observed experimentally, corresponding to guided modes of $\mathrm{CR}$ that bounces back and forth between the inner and outer walls of the shell. A more realistic description consisting of seven pores with an arrangement that mimics the one of the sample shown in Fig. 1(a) gives rise to similar results, confirming the mechanism of guided Cherenkov modes [solid curve in Fig. 2(a)]. A quantitative comparison with experiment has been carried out by convoluting the results from this latter model with the measured zero-loss 

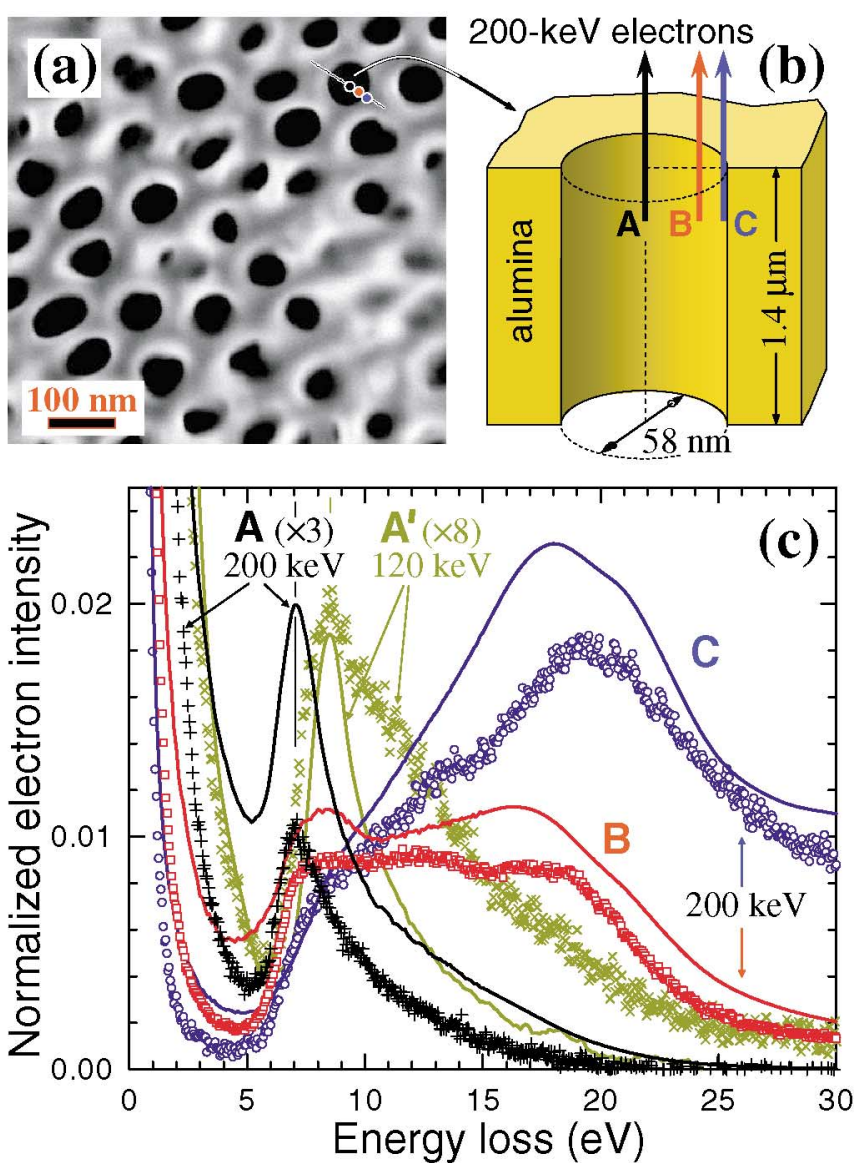

FIG. 1 (color). (a) STEM image of a $1.4-\mu \mathrm{m}$ thick porous alumina film showing the size and distribution of its pores, which run perpendicular to the film surfaces. (b) Schematic view of three different electron trajectories that cross the film through one of the pores. (c) Measured electron energy-loss spectra (EELS) for 200-keV electrons following the trajectories A-B-C considered in (b) (symbols), as compared with the present theory (curves). Results for $120-\mathrm{keV}$ electrons under axial trajectory are also shown (curve and symbols labeled $\mathrm{A}^{\prime}$ ). The calculated probability has been normalized such that the area under each curve is 1 a.u. and the heights of the experimental zero-loss peaks have been made equal to the theoretical ones. The theory has been convoluted with a measured $0.86-\mathrm{eV}$ FWHM elastic peak. The probability for spectra $\mathrm{A}$ and $\mathrm{A}^{\prime}$ has been multiplied by factors 3 and 8 , respectively, to improve readability.

peak and by integrating along the electron trajectory to allow for multiple losses [solid curves in Fig. 1(c)]. The agreement obtained without further adjustable parameters is excellent, especially if one realizes that absolute units are used in the vertical scale.

When more holes are considered, the loss spectra exhibit finer structures that converge eventually to the case of an infinite 2D crystal of triangular lattice, as shown in Fig. 2(b). However, these details cannot be resolved with the $\approx 1-\mathrm{eV}$ energy resolution of our experiment, and, furthermore, the lack of long-range order in our sample is not expected to be well represented by an ordered crystal. However, confined CR appears to emerge as

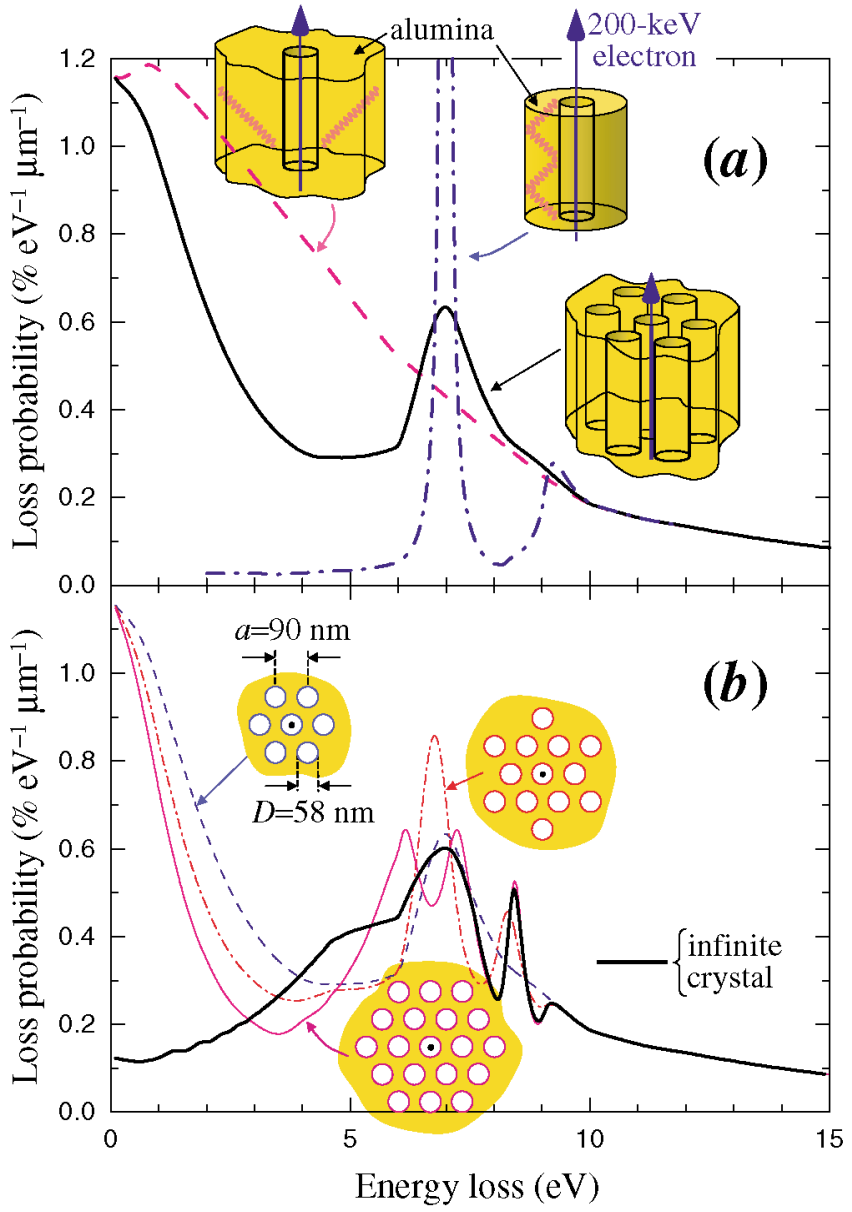

FIG. 2 (color). (a) Calculated EELS probability per unit length for $200-\mathrm{keV}$ electrons moving along the axis of a pore drilled in alumina when the pore is isolated (dashed curve), surrounded by six other pores (solid curve), or inside an alumina shell (dash-dotted curve). (b) Same as (a) for a pore surrounded by 6 pores (dashed curve), 12 pores (dash-dotted curve), 18 pores (thin solid curve), or and an infinite number of pores (thick solid curve), as shown in the insets. The pores are $58 \mathrm{~nm}$ in diameter and they are arranged in a triangular lattice with nearest-neighbor distance equal to $90 \mathrm{~nm}$ in all cases. The outer radius of the shell in (a) is $90 \mathrm{~nm}$.

pronounced structures near the $7-\mathrm{eV}$ region in all cases. Incidentally, the loss probability converges to the same value in the low-energy-loss limit for all finite geometries under discussion, which is an indication that CR cannot resolve finite pore structures in the long-wavelength limit. The infinite crystal, however, leads to a strong attenuation of Cherenkov losses in this region, which is consistent with the fact that lower energy losses require a larger number of cylinders to converge (i.e., they respond to a longer range of interaction).

Calculations for localized beams in infinite crystals have been carried out within a 2D version of the Korringa-Kohn-Rostoker multiple-scattering method [10]. However, band structure information has been derived from plane-wave expansion methods [11], which have also proved to be more convenient to simulate loss 


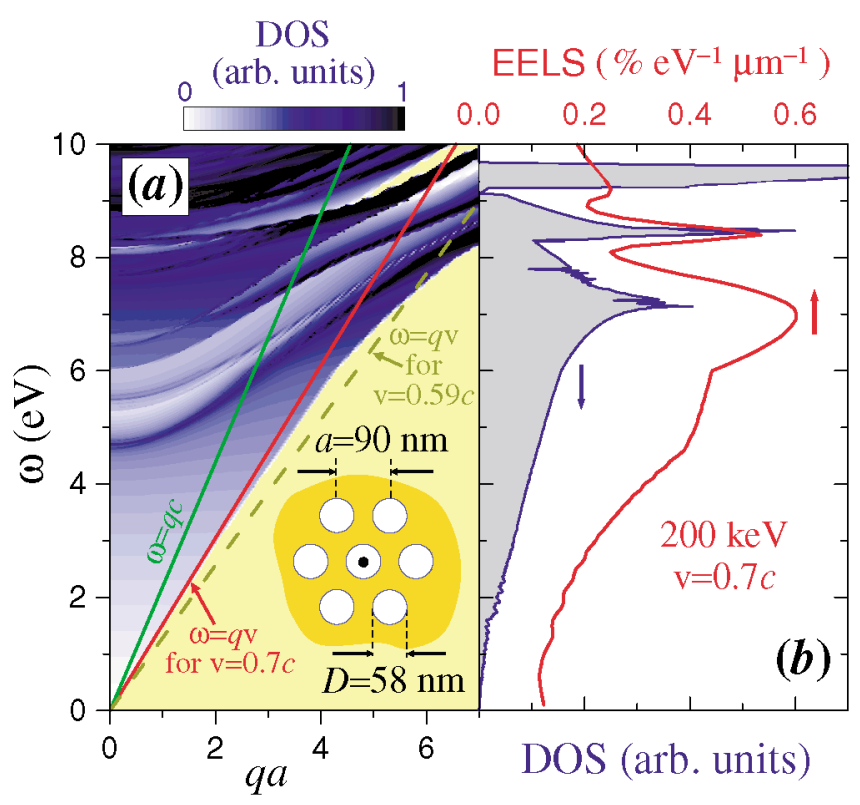

FIG. 3 (color). (a) Photonic DOS of a 2D crystal of cylindrical air holes drilled in alumina as a function of photon frequency $\omega$ and momentum component parallel to the cylinders, $q$. The crystal parameters are shown in the inset. (b) Calculated EELS probability for $200-\mathrm{keV}$ electrons $(v=$ $0.7 c$ ) moving along the axis of one of the pores in this crystal (red curve), as compared to the DOS along the $\omega=q v$ line [blue curve, taken from (a)].

probabilities in broad beams (see below), for which the average over all possible electron impact parameters has to be performed [12].
It should be noted that fast electrons moving with velocity $v$ along the cylinders ( $z$ direction) can be described by pointlike sources with space-time dependence $\delta(z-v t)=\sum_{q} \exp (i q z-i q v t)$, which permits establishing a relation between the frequency $\omega$ and the $z$ component of the momentum of the excited radiation allowed by the probe, $q$, namely, $\omega=q v$.

Further insight into the infinite crystal can be gained from the DOS, which is plotted in Fig. 3 as a function of photon energy $\omega$ and parallel momentum $q$. The kinematical constraint $\omega=q v$ [red line in Fig. 3(a) for 200$\mathrm{keV}$ electrons, that is, $v=0.7 c$ ] allows sampling regions of the $(q, \omega)$ plane below the light cone in vacuum (green line) that are not easily accessible using external light. The features of the DOS along $\omega=q v$ [shaded area in Fig. 3(b)] are reflected in the loss spectrum (red curve), although the similarity is not complete. When lower electron energies are considered, the intersection between the $\omega=q v$ line [e.g., the dashed line of Fig. 3(a) for 120$\mathrm{keV}$ electrons, that is, $v=0.59 c$ ] and the maxima of the DOS occurs at higher photon energies, and this explains the behavior observed in the measured loss spectrum for the axial trajectory using $120-\mathrm{keV}$ electrons [symbols labeled $\mathrm{A}^{\prime}$ in Fig. 1(c)], which shows a prominent 8.3-eV feature that is well reproduced by our theory [curve labeled $\mathrm{A}^{\prime}$ in Fig. 1(c)].

A more detailed examination of the relation between EELS and photonic band structures is illustrated in Figs. 4 and 5 for a triangular 2D crystal of air holes drilled in $\mathrm{Si}$ $(\epsilon=11.9)$ with a diameter to lattice constant ratio $D / a=0.8$. Photonic bands are shown in Fig. 4(a) over

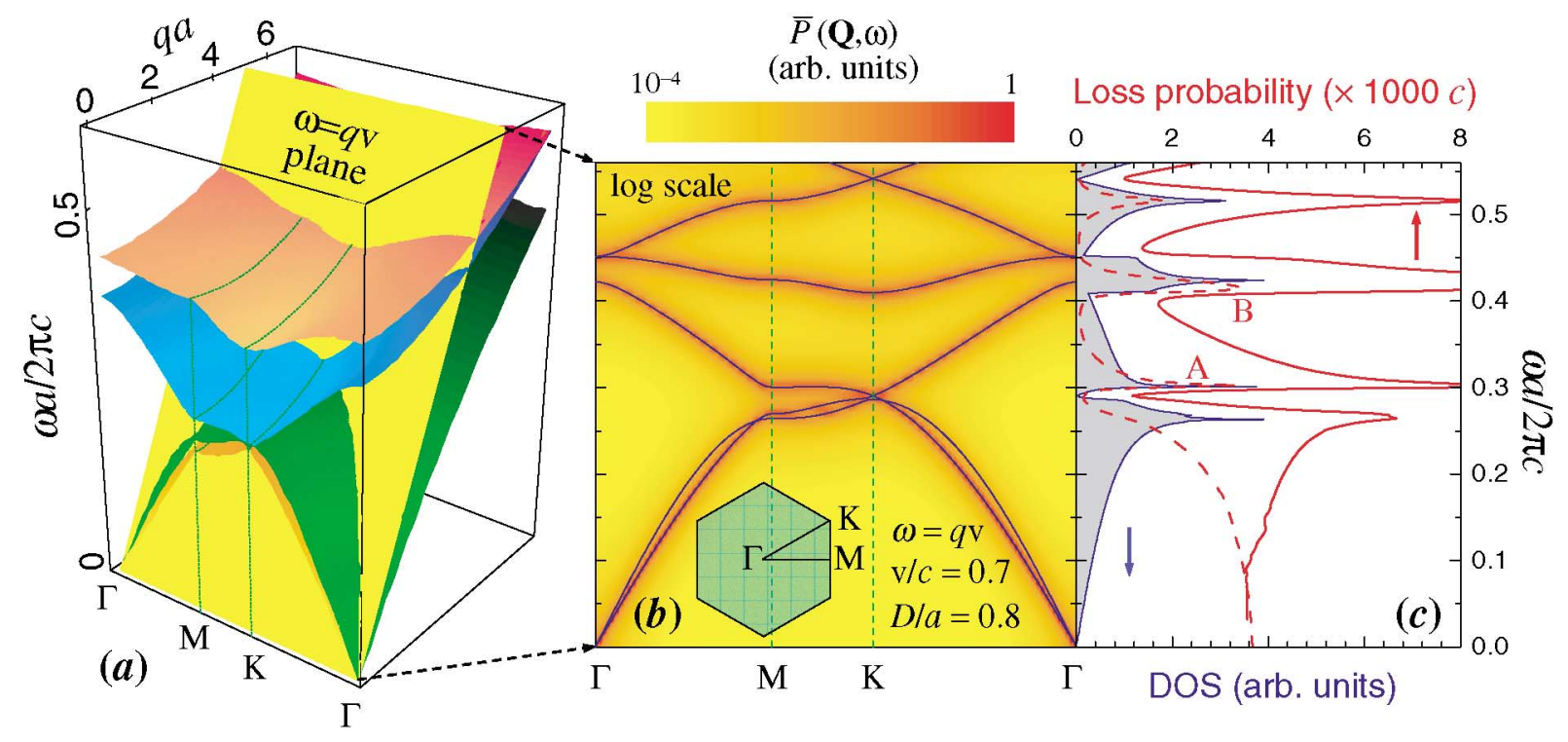

FIG. 4 (color). (a) Photonic band structure of a triangular 2D crystal of cylindrical air holes drilled in Si $(\epsilon=11.9)$ for a diameter to lattice constant ratio $D / a=0.8$. Only the four lowest bands are shown for the sake of clarity. (b) Intersection of these bands with the $\omega=q v$ plane for $v=0.7 c$ (blue curves). The underlying density plot (in log scale) shows the calculated EELS probability averaged over electron impact parameters, $\bar{P}(\mathbf{Q}, \omega)$, and resolved in both photon frequency $\omega$ and momentum transfer perpendicular to the cylinders axes, $\mathbf{Q}$. (c) DOS along $\omega=q v$ (shaded area) as compared with calculated loss spectra for an axial electron trajectory (broken curve) and for the average over impact parameters (red solid curve). 


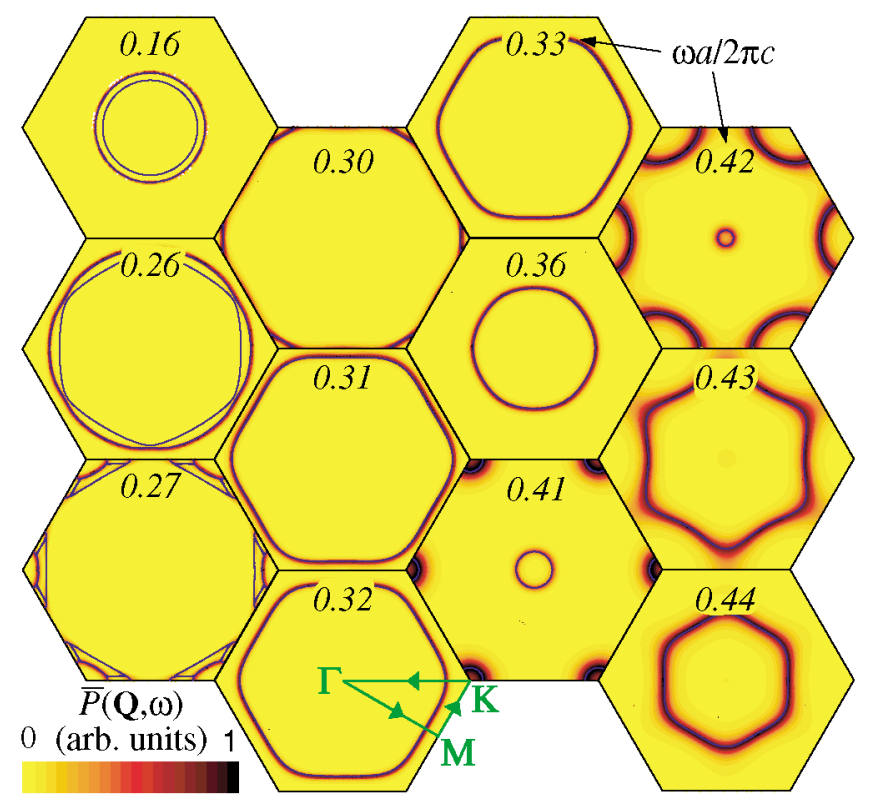

FIG. 5 (color). Photonic band structure (blue curves) compared with the EELS probability $\bar{P}(\mathbf{Q}, \omega)$ (density plot) under the same conditions as in Fig. 4. Each hexagon represents the first Brillouin zone for a given value of $\omega$, as shown by labels.

a wide range of $q$ 's. The intersection with the $\omega=q v$ plane (in yellow) is represented in Fig. 4(b) (blue curves) as compared with the EELS probability for a broad electron beam, $\bar{P}(\mathbf{Q}, \omega)$, resolved in lost energy $\omega$ and momentum transfer perpendicular to the trajectory, $\mathbf{Q}$ (density plot). $\bar{P}(\mathbf{Q}, \omega)$ stands for the probability that the electron loses energy $\omega$ while it acquires a momentum $\mathbf{Q}$ perpendicular to its velocity, which manifests itself as a deflection of the electron trajectory after scattering. $\bar{P}(\mathbf{Q}, \omega)$ can be measured from the angular distribution of electrons passing through the crystal for selected energy losses, as it has been done in the past for electrons transmitted through thin films [13]. A different sampling of the band structure is offered in Fig. 5, where each hexagon represents the first Brillouin zone for fixed values of $\omega$. Most of the bands (blue curves) are reproduced by the maxima of $\bar{P}(\mathbf{Q}, \omega)$ in these figures (red regions), except for the second lowest band, which goes largely unnoticed by the electrons, since it has a dominant $\mathrm{H}$ polarization character [11] that is orthogonal to the electric field of the probe [12].

The projection of the DOS on $\omega=q v$ is compared with the loss probability in Fig. 4(c). Some of the features of the DOS (shaded area) are reproduced by loss spectra both for localized trajectories (dashed curve) and for broad beams (red solid curve), including gaps in the projected DOS, which show up as dips in the EELS probability, indicating that no radiation modes are available to be excited. Moreover, peak A originates in con- tributions from the third band near $M$ points [see Fig. 4(b)], and it lies on top of a maximum in the DOS, unlike peak B for the axial trajectory, which occurs near a sudden change in the DOS. Actually, peak B comes from coupling to regions around $\mathrm{K}$ points where the fourth band is nearly flat, according to Fig. 4(b). This is also seen in Fig. 5 for $\omega a / 2 \pi c=0.41$. Indeed, peaks A and B originate in saddle points of the $2 \mathrm{D}$ bands, which give rise to logarithmic Van Hove singularities in the DOS [14] (transmitted to the loss spectra, since the electrons can couple to a larger number of photonic modes around these frequencies), whereas the discontinuities in the DOS, which show up as sudden changes in the loss probability, arise from maxima and minima in these bands.

In conclusion, we have shown that fast electrons can be used for directly probing photonic crystal band structures. Our hope is that the excellent agreement obtained between theory and experiment for porous alumina and the reported mapping of photonic bands by momentumresolved Cherenkov losses can stimulate further experimental work on EELS as a tool for investigating photonic crystals.

[1] E. D. Palik, Handbook of Optical Constants of Solids (Academic Press, New York, 1985).

[2] D. Ugarte, C. Colliex, and P. Trebbia, Phys. Rev. B 45, 4332 (1992).

[3] P. A. Cherenkov, Dokl. Akad. Nauk SSSR Ser. A 2, 451 (1934); I. M. Frank and I. Tamm, Dokl. Akad. Nauk SSSR Ser. A 14, 109 (1937).

[4] S. J. Smith and E. M. Purcell, Phys. Rev. 92, 1069 (1953); see F. J. García de Abajo and L. A. Blanco, Phys. Rev. B 67, 125108 (2003) for similar effects in 3D photonic crystals.

[5] T. E. Stevens, J. K. Wahlstrand, J. Kuhl, and R. Merlin, Science 291, 627 (2001).

[6] C. Luo, M. Ibanescu, S. G. Johnson, and J. D. Joannopoulos, Science 299, 368 (2003).

[7] D. Crouse, Y.-H. Lo, A. E. Miller, and M. Crouse, Appl. Phys. Lett. 76, 49 (2000).

[8] A. G. Pattantyus-Abraham, N. Zabala, A. Rivacoba, F. J. García de Abajo, and M. O. Wolf (to be published).

[9] F. J. García de Abajo and A. Howie, Phys. Rev. Lett. 80, 5180 (1998); Phys. Rev. B 65, 115418 (2002).

[10] K. M. Leung and Y. Qiu, Phys. Rev. B 48, 7767 (1993).

[11] K. M. Ho, C. T. Chan, and C. M. Soukoulis, Phys. Rev. Lett. 65, 3152 (1990); K. Busch and S. John, Phys. Rev. E 58, 3896 (1998).

[12] F. J. García de Abajo, A. Rivacoba, N. Zabala, and P. M. Echenique, Phys. Rev. B (to be published).

[13] C. H. Chen and J. Silcox, Phys. Rev. Lett. 35, 390 (1975).

[14] L. Van Hove, Phys. Rev. 89, 1189 (1953); N.W. Ashcroft and N. D. Mermin, Solid State Physics (Saunders College Publishing, New York, 1976). 